\section{Primary Adenocarcinoma of the Lacrimal Gland}

Sang-Oon Baek, Yoon-Jae Lee, Suk-Ho Moon, Young-Jin Kim, Young-Joon Jun

Department of Plastic and Reconstructive Surgery, Bucheon St. Mary's Hospital, The Catholic University of Korea, Bucheon, Korea

Correspondence: Young-Joon Jun

Department of Plastic Surgery, Bucheon St. Mary's Hospital, The Catholic

University of Korea, 327 Sosa-ro, 327 beon-gil, Wonmi-gu, Bucheon 420-717, Korea

Tel: +82-32-340-2095, Fax: +82-32-340-2666

E-mail:psdoc@korea.com

No potential conflict of interest relevant to this article was reported.

Received: 26 Jun 2012 • Revised: 12 Aug 2012 • Accepted: 14 Aug 2012 pISSN: 2234-6163・ elSSN: 2234-6171

http://dx.doi.org/10.5999/aps.2012.39.5.578 • Arch Plast Surg 2012;39:578-580

Copyright (C) 2012 The Korean Society of Plastic and Reconstructive Surgeons This is an Open Access article distributed under the terms of the Creative Commons. Attribution Non-Commercial License (http://creativecommons.org/licenses/by-nc/3.0/) which permits unrestricted non-commercial use, distribution, and reproduction in any medium, provided the original work is properly cited.

Tumors originating from the lacrimal gland account for approximately $10 \%$ of orbital tumors and have a large diversity of anatomic and pathological types. Among these, malignant epithelial tumors of the lac- rimal gland are extremely rare, constituting less than $5 \%$ of all orbital lesions [1]. Adenocarcinoma of the lacrimal gland arises in the upper eyelid and usually presents as a mass accompanied by symptoms such as pseudoptosis, exophthalmos, dystopia, pain, and reduced visual acuity $[1]$. However, patients with this disease are often symptomless, leading them to be diagnosed at the advanced stage. Moreover, on radiologic examination, it frequently presents as a cystic mass and is often misdiagnosed as a benign tumor. In this report, we present a case of a patient with rare disease of primary adenocarcinoma originating from the lacrimal gland.

A 50-year-old male patient was transferred from the ophthalmology department to plastic surgery department for blepharoptosis, hypotropia, and exophthalmos of his right eye. These symptoms had developed progressively over the previous 6 months. Initially, the patient complained of no pain, while the ophthalmologic and laboratory tests revealed no definite abnormalities. On physical examination, we observed proptosis, dystopia, and ptosis of his right eye (Fig. 1).

An MRI scan of the ocular space revealed a

$2.3 \times 2.2 \mathrm{~cm}$ benign cystic tumor on the right side of the lacrimal gland (Fig. 2). With the impression of a benign tumor, an excisional biopsy was carried out through the subbrow excision and a well demarcated cystic mass was observed intraoperatively. The mass was confirmed to be primary adenocarcinoma on pathologic examination (Fig. 3).

On immunohistochemistry examination, cytokeratin 7 was positive, whereas cytokeratin 20 turned out to be negative. These results were adequate enough to conclude that the malignant epithelial tumor had

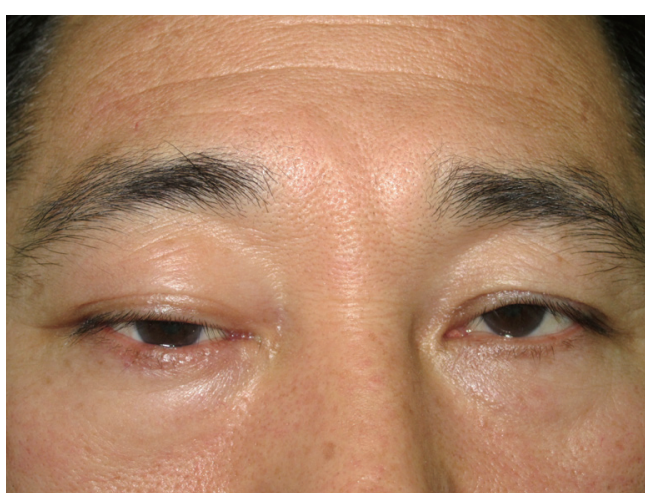

Fig. 1. Initial photograph shows right blepharoptosis, hypotropia, and exopthalmos. 
originated from the lacrimal gland [2]. Full body positron emission tomography-computed tomography (PET-CT) revealed no regional or distant metastasis (Fig. 4).

Generally, the treatment for primary adenocarcinoma on the lacrimal gland is wide surgical excision of the tumor with orbital bone resection and eyeball enucleation, followed by radiotherapy. Nevertheless, this patient somehow refused the additional surgery due to repulsion to major surgery. High dose radiotherapy and anti-cancer treatment was carried out instead (Fig. 5).

The lacrimal gland is considered in some ways to

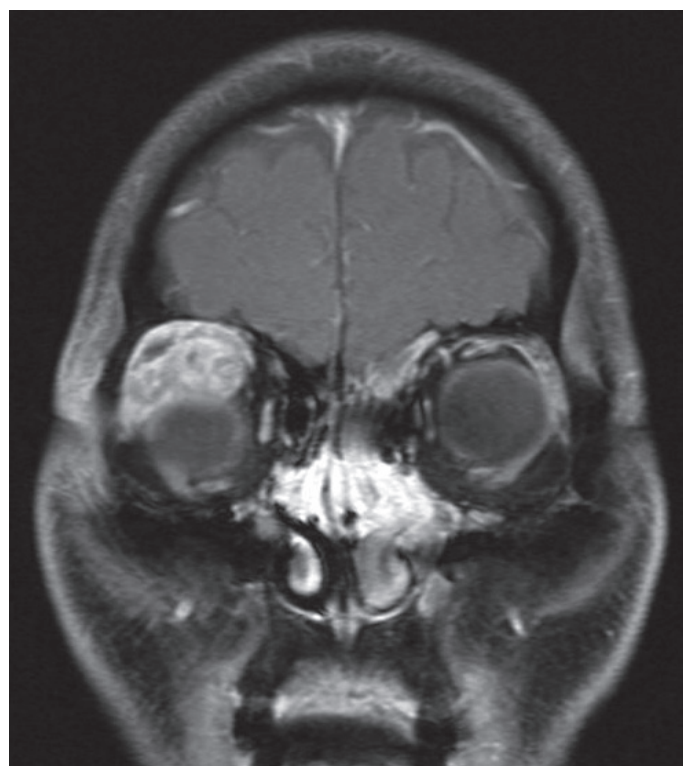

be a type of minor salivary gland that shares histologic features with the major salivary glands. Hence, in many respects, lacrimal gland tumors are similar to those that arise in the major salivary glands.

According to a clinical series from the Wills Eye Hospital, lacrimal gland tumors represent almost $10 \%$ of the orbital lesions, with epithelial lesions accounting for $20 \%$ of them. Among epithelial lesions, $55 \%$ are benign and $45 \%$ are malignant. Furthermore, among the malignancies, adenoid cystic carcinoma is most frequently found (66\%), followed by carcinoma ex pleomorphic adenoma (18\%), primary adenocar-
Fig. 2.

An magnetic resonance imaging image of the eye revealed a $2.3 \times 2.2 \mathrm{~cm}$ cystic tumor on the right side of the lacrimal gland.

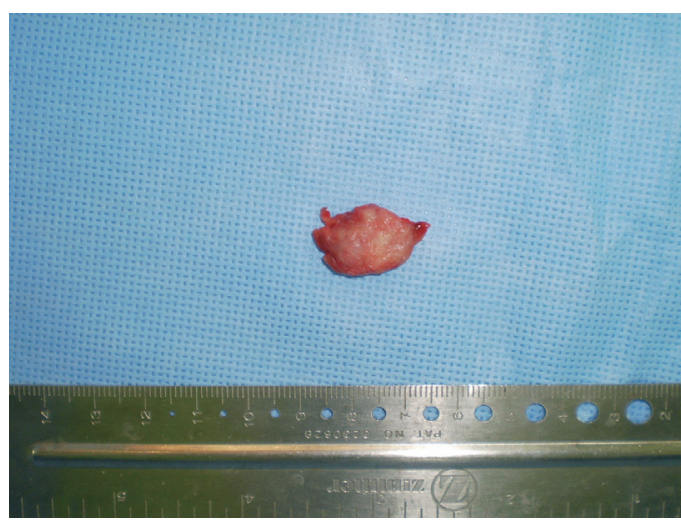

Fig. 3.

Excised mass. Gross examination revealed a well demarcated, ovalshaped mass, measuring $2.0 \times 1.5 \times 1.0 \mathrm{~cm}$.

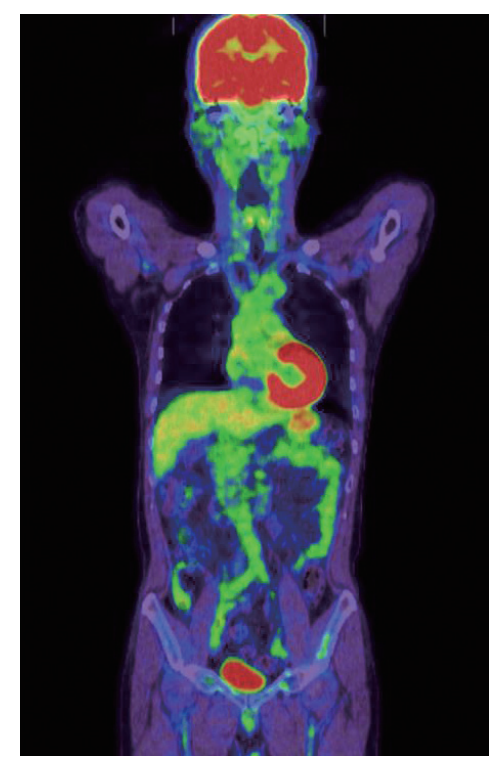

Fig. 4.

A full body positron emission tomographycomputed tomography showed no abnormal metastasis to other organs.

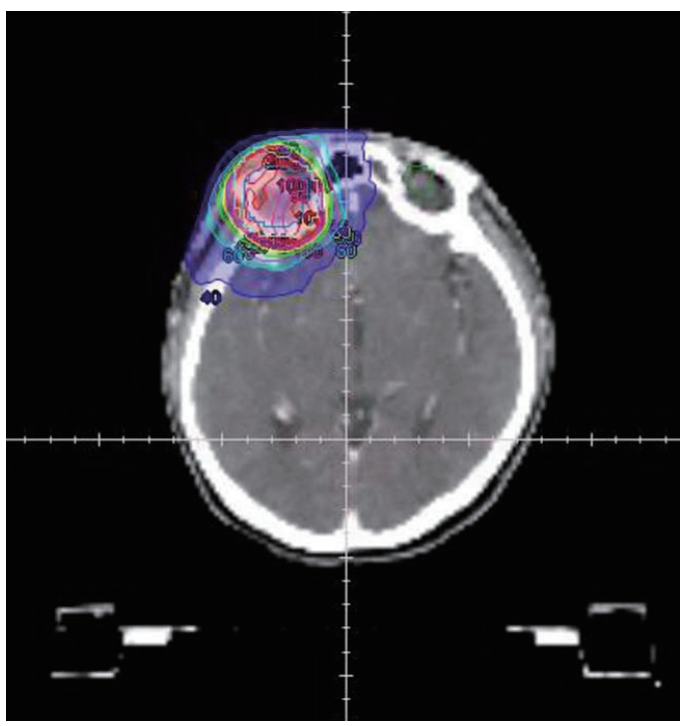

Fig. 5.

A full body positron emission tomography-computed tomography showed no abnormal metastasis to other organs. 
cinoma (9\%) and mucoepidermoid carcinoma (3\%), in that order [3].

Patients with primary adenocarcinoma of the lacrimal gland present with a protruding mass in the upper eyelid accompanied by symptoms such as exophthalmos, pseudoptosis, dystopia, and in advanced disease, even reduced visual acuity [1]. Pain can be secondary to involvement of bone or nerve, and is often an important sign suggesting malignancy. However, benign lacrimal gland tumors can also sometimes induce mild pain and hence, pain alone cannot be a single indicator suggestive of malignancy. Symptoms such as blepharoptosis, ocular motility disturbance, and diplopia are more likely to occur in malignant tumors. Other signs suggestive of malignancy include bone erosion on radiological studies and rapid growth in size. The patient introduced in this case presented with symptoms such as blepharoptosis, hypotropia, and exophthalmos, which can be possibly present in lacrimal gland malignancies. However, the patient did not complain of any pain or other typical clinical symptoms that could strongly suggest malignancy.

The duration of the symptoms in patients with malignant lacrimal gland tumors is usually shorter than that of benign lesions, usually less than 6 months.

The clinical diagnosis of malignant lacrimal gland tumor is suspected based on the signs and symptoms mentioned earlier. The most important diagnostic tools are computed tomography (CT) and magnetic resonance imaging (MRI). These studies generally demonstrate a round, ovoid, or elongated soft tissue mass that sometimes shows an irregular outline. These tumors, however, are frequently misdiagnosed as benign in nature.

Due to the infrequent occurrence of this disease, no clear protocol for treatment of primary adenocarcinoma yet exists. Many studies have attempted to establish the treatment protocol, but controversies continue in the medical literature without clear guidelines. The histopathology categorizes primary adenocarcinoma into low- and high-grade lesions, and together with the immunohistochemistry examination, this classification allows for a precise diagnosis and appropriate management of such rare lacrimal gland lesions.

In the majority cases of lacrimal gland malignancies, the physicians need to communicate with the patients to determine the optimal treatment plan with the goal of complete removal of the tumor, orbital bone resection, and maintaining health and intact vision of the patient's eye. If incompletely removed, the recurrence rate is high [3] and the disease can progress rapidly. Thus, it has been recommended that radical excision of the lesion is essential to prevent such recurrence or progression [4]. An en bloc resection of the orbital tumor and contents, together with orbitectomy, is considered to be the most logical and effective method of treatment [1]. Recent studies have also proposed that adjuvant radiotherapy and/ or chemotherapy could be effective in preventing the recurrence. It is believed that the combination of cisplatin and doxorubicin induces tumor cell necrosis and reduction in tumor size [5].

Primary adenocarcinoma in the lacrimal glands is extremely rare. Symptoms include blepharoptosis, dystopia, eyeball deviation, and exophthalmos. It is important to consider the possibility of malignant tumor in patients with such symptoms, in that an early radical excision is mandatory. CT or MRI is a helpful ancillary diagnostic tool in determining the size and location of the tumor. However, these radiologic examinations frequently present the tumor as a welldemarcated cystic lesion misleading the examiner into diagnosing it as benign. With the final precise diagnosis, radical surgery such as orbital enucleation followed by orbital reconstruction, with high dose adjuvant chemotherapy or radiotherapy should be carried out.

\section{References}

1. Clauser L, Galie M, Tieghi R, et al. Adenocarcinoma of the lacrimal gland: report of a case. J Oral Maxillofac Surg 2002;60:318-21.

2. Damasceno RW, Holbach LM. Primary ductal adenocarcinoma of the lacrimal gland: case report. Arq Bras Oftalmol 2012;75:64-6.

3. Bernardini FP, Devoto MH, Croxatto JO. Epithelial tumors of the lacrimal gland: an update. Curr Opin Ophthalmol 2008;19:409-13.

4. Janecka I, Housepian E, Trokel S, et al. Surgical management of malignant tumors of the lacrimal gland. Am J Surg. 1984;148:539-41.

5. Meldrum ML, Tse DT, Benedetto P. Neoadjuvant intracarotid chemotherapy for treatment of advanced adenocystic carcinoma of the lacrimal gland. Arch Ophthalmol 1998;116:315-21. 